\title{
Some Problems of Relativistic Thermodynamics Allowing for Primordial Substance Anisotropy
}

\author{
E.V.Veitsman \\ Veitsman's Science Project, 28 Apartment, 5 Klimashkin Str., Russia
}

Copyright $@ 2015$ Horizon Research Publishing All rights reserved.

\begin{abstract}
Some processes of heat and electrical charge transfer under relativistic conditions have been studied allowing for primordial substance anisotropy. In particular, electrical charge convection and conductivity were examined in the framework of 3-D and 4-D formalisms. Dependences were obtained in which the left-hand-sides and right-hand-sides transformed identically as $\mathbf{v} \rightarrow c$, where $\mathbf{v}$ was the velocity of the object under study, $c$ was the speed of light. Dependences were also obtained for the heat transfer in the framework of 4-D formalism under relativistic conditions when the substance in the system was primordially anisotropic. The above dependences for the charge transfer are correct for systems where charges, e.g., electrons, can move more quickly than photons (Cherenkov's case). A consistent relativistic thermodynamics was also to be obtainable only if H.Ott's temperature transformation under relativistic conditions takes place. Range of Considered Symbols. $J^{\alpha}$ and $J_{\alpha}$ are the contravariant and covariant 4-current density; $j^{\alpha}$ is the 3 -current density; $E_{x}, E_{y}, E_{z}$ are the tensor components of the electrical field intensity. $B_{x}, B_{y}, B_{z}$ are the tensor components of the magnetic field intensity; $\sigma$ is the conductivity; $\rho$ is the charge density; $u^{\alpha}, u_{\beta}$ are the contravariant and covariant dimensional velocities; $\sigma_{\alpha \beta}\left(L_{\alpha \beta}\right)$ is the conductivity tensor; $T$ is the temperature; $\Delta l_{\alpha(\beta)}$ are the vectors oriented in space; $s$ is the invariant interval; $G_{\beta}$ and $G^{\alpha}$ are the covariant and contravariant 3D-electrical intensity; $E_{\beta}$ and $E^{\beta}$ are the covariant and contravariant 4D-electrical intensity; $q$ is the quantity of heat which is allocating in unit of volume per unit of time $\left(\mathrm{J} \cdot \mathrm{cm}^{-3} \cdot \mathrm{s}^{-1}\right) ; \Lambda^{\alpha \beta}$ and $\Lambda_{\alpha \beta}$ are the contravariant and covariant 4-tensor of conductivity; $I_{(\lambda)}^{\alpha}$ is the heat flux contravariant $4 \mathrm{D}$-vector; $D_{(\lambda)}^{\varepsilon \beta}$ is the heat flux coefficient (contravariant 4-tensor).
\end{abstract}

Keywords Relativistic Thermodynamics, Heat Transfer,
Charge Transfer, Conductivity, Relativistic Temperature, Convection Charge Transfer, Joule Heat, Cherenkov's Case

PACS: 03.30.+p , 05.70.-a , 05.60.-k

\section{Introduction}

In [1] transfer processes under relativistic conditions were studied. They were the processes of heat and mass transfer and the viscous flow process. The medium was primordially isotropic. The transfer of electrical charges was not studied. The dependences over the velocity interval $0-\mathbf{v}$ were obtained. The left and right sides of the dependences transform identically under the Lorentz transformations. The kinetic coefficients therein should be tensors of rank 2 . The above results are correct for the case represented in Fig.1, i.e., for

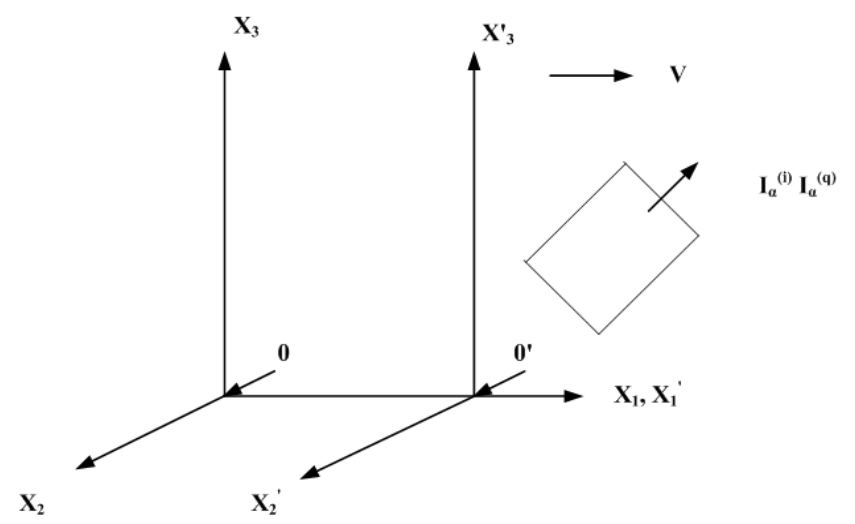

Figure 1. $\mathrm{X}_{1}, \mathrm{X}_{2}, \mathrm{X}_{3}$ and $\mathrm{X}_{1}^{\prime}, \mathrm{X}_{2}^{\prime}, \mathrm{X}_{3}^{\prime}$ are reference frames at rest and moving with a velocity $\mathbf{v}$ respectively; $\mathrm{I}_{\alpha}{ }^{(\mathrm{i})}$ and $\mathrm{I}_{\alpha}{ }^{(\mathrm{q})}(\alpha=1,2,3)$ are electrical charge and heat fluxes through a unit surface.

Before E.V.Veitsman, some results were obtained by C.Eckart [2] and L.D.Landau with E.M.Lifshitz [3] for the heat and mass transfer in the system represented in Fig.1 as well. Eckart also obtained in [2] a dependence for electrical charge transfer as follows:

$$
J^{\alpha}=\rho u^{\alpha}-\sigma F^{\alpha \beta} u_{\beta},
$$




$$
F^{\alpha \beta}=\left(\begin{array}{cccc}
0 & -E_{x} & -E_{y} & -E_{z} \\
E_{x} & 0 & -B_{z} & B_{y} \\
E_{y} & B_{z} & 0 & -B_{x} \\
E_{z} & -B_{y} & B_{x} & 0
\end{array}\right)
$$

where $J^{\alpha}$ is the current density $\left(\mathrm{C} \cdot \mathrm{m}^{-2} \cdot \mathrm{s}^{-1}\right) ; \sigma$ is the conductivity $\left[\mathrm{A} \cdot \mathrm{V}^{-1} \cdot \mathrm{m}^{-1}(\mathrm{SI}) ; \mathrm{s}^{-1}\right.$ (Gaussian system) $] ; F^{\alpha \beta}$ is the electromagnetic field tensor; $E_{x}, E_{y}, E_{z}$ are the tensor components of the electrical field intensity $\left(\mathrm{V} \cdot \mathrm{m}^{-1}\right.$; $\left.\mathrm{g}^{1 / 2} \cdot \mathrm{cm}^{-1 / 2} \cdot \mathrm{s}^{-1}\right) ; B_{x}, B_{y} B_{z}$ are the tensor components of the magnetic field intensity $\left(\mathrm{A} \cdot \mathrm{m}^{-1} ; \mathrm{g}^{1 / 2} \cdot \mathrm{cm}^{-1 / 2} \cdot \mathrm{s}^{-1}\right) ; \rho$ is a charge density $\left(\mathrm{C} \cdot \mathrm{cm}^{-3}\right) ; u^{\alpha}, u_{\beta}$ are the contravariant and covariant dimensional velocities. Evidently, $E^{\alpha}=F^{\alpha \beta} u_{\alpha}$ and expression (1) are incorrect for a primordially anisotropic medium where $\sigma$ is the tensor of rank 2, i.e., $\sigma_{\alpha \beta}$.

In fact for this case $J_{\alpha}=J\left(\rho, \sigma E_{x}, \sigma E_{y}, \sigma E_{z}\right)$ if, according to Eckart, the medium in the object is isotropic. In our opinion, the medium will not always be isotropic in the moving object for the observer being in the laboratory reference frame. Then the quantity $\sigma$ can be a tensor of rank 2 . As we shall see below, the situation will be more complex for a primordially anisotropic medium.

The correctness of the expressions (1) is very doubtful. Indeed, first, will their left-hand-side and right-hand-side transform identically under relativistic conditions? Second, the dimension of $J_{\alpha}$ is differs from the dimension of the first term on the right-hand-side (1) unless $\rho u^{\alpha}$ multiplied by "c". At last, for an observer being in the laboratory reference frame the conductivity $\sigma$ must be a tensor of rank 2 like the heat transition coefficient $D_{i j}^{(\lambda)}$ see [1]).

The heat transfer is represented by Eckart as

$$
\begin{gathered}
q^{\alpha}=k S^{\alpha \beta}\left[\left(d \theta / d x^{\beta}\right)+\theta D u_{\beta}\right], \\
S^{\alpha \beta}=g^{\alpha \beta}+u^{\alpha} u^{\beta},
\end{gathered}
$$

where $q^{\alpha}$ is the heat flux $\left(\mathrm{J} \cdot \mathrm{cm}^{-2} \cdot \mathrm{s}^{-1}\right) ; k$ is the heat transfer coefficient $\left(\mathrm{J} \cdot \mathrm{cm}^{-1} \cdot \operatorname{grad}^{-1}\right) ; g^{\alpha \beta}$ is the contravariant fundamental tensor; $D \theta u_{\beta}$ is a relativistic component; it has no classical analogy; it "implies as isothermal flow of heat in accelerated matter, in the direction opposite to the acceleration. It is ordinarily small and may be explained as due to the inertial of energy"[2]. The coefficient $k$ is a scalar.

At last, following Richard Tolman, K.Eckart considers that $T^{0} \equiv \theta,\left(\theta / \theta_{0}\right)=$ const, i.e., the temperature is a relativistic invariant, which is also incorrect.

L.D.Landau and E.M.Lifshitz obtained the following expression for the heat transfer in Euclidean space under relativistic conditions:

$$
\boldsymbol{q}=-\kappa\left(\nabla T-\frac{T}{w} \nabla p\right),
$$

where $\boldsymbol{q}$ is the vector of the heat flux $\left(\mathrm{J} \cdot \mathrm{cm}^{-2} \cdot \mathrm{s}^{-1}\right) ; w$ is a heat function $\left(\mathrm{J} \cdot \mathrm{cm}^{-3}\right) ; p$ is the pressure; $\kappa$ is the heat transfer coefficient $\left(\mathrm{J} \cdot \mathrm{cm}^{-1} \cdot \mathrm{s}^{-1} \cdot \operatorname{grad}^{-1}\right)$.

According to Landau and Lifshitz, the second term on the right side of (4) is of purely relativistic nature, like the second term on the right side of (3). Like this term, it is also very small in magnitude as compared with the first one on the right side of (3) taking into consideration that $w=e+p$; $e=m c^{2}$. The temperature $T$ is independent of the system velocity for the observer being in the laboratory reference frame. The coefficient $\kappa$ is a scalar quantity, i.e., according to the authors [3], the medium in the system is isotropic for the above observer. However, it is incorrect (see [1]). Besides, the left and right sides of (4) will transform differently under relativistic conditions.

In [1] E.V.Veitsman obtains, in turn, an expression for the heat transfer $I_{\alpha}^{(q)}\left(\mathrm{J} \cdot \mathrm{cm}^{-2} \cdot \mathrm{s}^{-1}\right)$ in Euclidean space under relativistic conditions for the observer being in the laboratory reference frame:

$$
I_{\alpha}^{(q)}=-D_{\alpha \beta}^{(q)} \frac{\partial q}{\partial x_{\beta}}, D_{\alpha \beta}^{(q)}=0, \alpha \neq \beta,
$$

where $q$ is the heat density at the point $x_{\beta}\left(\mathrm{J} \cdot \mathrm{cm}^{-3}\right) ; D_{\alpha \beta}^{(q)}$ is the so-called thermal diffusion coefficient $(\mathrm{cm} 2 \cdot \mathrm{s}-1)$.

Expression (5) is correct in case the medium in our system is primordially isotropic. At last, the left and right sides of (5) transform identically under relativistic conditions. However, will expression (5) be correct if the medium primordially anisotropic? In this case, the heat transfer process should be described in such a way:

$$
I_{\alpha}^{(q)}=-D_{\alpha \beta}^{(q)} \frac{\partial q}{\partial x_{\beta}},
$$

i.e., the coefficient $D_{\alpha \beta}^{(q)}$ in (6) may be non-zero for crystals if $\alpha \neq \beta$ [4].

If in (5) and (6) we replace quantity q by $\rho$, then we shall obtain an expression for the electron diffusion. It will be correct under relativistic conditions if the medium is primordially isotropic. Will it be correct if the media is primordially anisotropic?

Analogous picture may take place for charge transfer when the coefficient $\sigma$ could be as tensor of rank 2, i.e., $\sigma_{\alpha \beta} \neq 0$, if $\alpha \neq \beta$. In this case the expression for the density current $j_{\alpha}\left(\mathrm{g}^{1 / 2} \cdot \mathrm{cm}^{-1 / 2} \cdot \mathrm{s}^{-2}\right)$ will have such a form in Euclidean space for the observer being in the laboratory reference frame:

$$
j_{\alpha}=\sigma_{\alpha \beta} G_{\beta}, \alpha, \beta=1,2,3,
$$


where $\sigma_{\alpha \beta}$ is the conductivity tensor, $G_{\beta}$ is the electrical intensity.

Dependence (7) is Lorentz invariant in 3D-space when medium in the system is primordially isotropic: the left and right sides of (7) transform identically as $v \rightarrow c$; c is the speed of light. Namely, the dimensions of the quantities $j_{\alpha}, \sigma_{\alpha \beta}$ and $G_{\beta}$ are $\mathrm{C} \cdot \mathrm{cm}^{-2} \cdot \mathrm{s}^{-1}, \mathrm{C} \cdot \mathrm{cm}^{-1} \cdot \mathrm{V}^{-1} \cdot \mathrm{s}^{-1}$ and $\mathrm{V} \cdot \mathrm{cm}^{-1}$. $\mathrm{C} \neq \mathrm{C}(v), \quad t=t_{0} / \sqrt{1-\beta^{2}}, \quad l=l_{0} \sqrt{1-\beta^{2}} \quad$ in the international system of units. As to the dimension of the quantity $\mathrm{V}$, it is not here taken into account since a voltage disappears from the right side of (7) due to its reduction. Will (7) be correct if the medium is primordially anisotropic?

If we take current intensity $I\left(\mathrm{~A}=\mathrm{C} \cdot \mathrm{s}^{-1}\right)$ instead its density $j_{\alpha}$, then Ohm's law will take the following form for an isotropic body [4]:

$$
I=\frac{U}{R}=\sigma U,
$$

Where $U$ is the electric potential difference (V): $R$ is the electrical resistance (V/A).

Formula (8) will be correct if the quantity $U$ is not very large. As seen, the left and right sides of (8) will transform identically as $v \rightarrow c$.

At last, we must concern H.Ott's famous work [5]. He studied, in particular, the processes of electrical charge transfer under relativistic conditions as well. The medium is isotropic, therefore Ott represents the processes of charge transfer and Joule heat as

$$
\begin{gathered}
j_{\alpha}=\sigma E_{\alpha}, \\
q=\boldsymbol{j} \cdot \boldsymbol{E}=j_{x} E_{x}+j_{y} E_{y}+j_{z} E_{z},
\end{gathered}
$$

where $j_{\alpha}$ is the current density $\left[\mathrm{C} \cdot \mathrm{cm}^{-2} \cdot \mathrm{s}^{-1}(\mathrm{SI}) ; \mathrm{g}^{1 / 2} \cdot \mathrm{cm}^{-1 / 2}\right.$. $\mathrm{s}^{-2}$ (Gaussian Units)]; $\boldsymbol{E}$ is the electric intensity $\left(\mathrm{g}^{1 / 2} \cdot \mathrm{cm}^{-1 / 2}\right.$. $\mathrm{s}-2) ; \sigma$ is the specific electrical conductivity (s-1); $q$ is the quantity of heat which is allocating in unit of volume of the conductor per unit of time when the conductor carries a current

$$
\left(\mathrm{J} \cdot \mathrm{cm}^{-3} \cdot \mathrm{s}^{-1}\right)
$$

H.Ott shows, in particular, that the temperature $\mathrm{T}$ in the system varies under relativistic conditions for the observer in the reference frame as

$$
T=T_{0} / \sqrt{1-\beta^{2}},
$$

hereinafter the subscript " 0 " denotes a quantity in the reference frame at rest.

Meanwhile, Max Planck considered that $T=T_{0} \sqrt{1-\beta^{2}}$ (see, e.g., [6]). Here we should note as well that in the courses of relativistic electrodynamics the problem of the temperature transformation under relativistic conditions is not studied (see, e.g., [7]). In turn,
E.V.Veitsman, studying the transformation of some physical parameters under relativistic conditions, comes, in particular, to a conclusion that the temperature must transform according to relation (11) [8-11].

As seen, Eckart, Landau with Lifshitz and Ott study systems, considering that medium therein is isotropic for the observer from the laboratory reference frame. The researchers are sure that the dependences they obtained are correct under relativistic conditions. Finally, they did not study objects in the medium being primordially anisotropic. Therefore we can now formulate the main goal of this work. First, will the relation (6) be correct for the observer in the laboratory reference frame (3-D formalism)? Second, we contemplate obtaining an expression for the heat transfer in Minkowski space (4-D formalism). The third, we consider obtaining expressions for the electric charge transfer in Euclidean and Minkowski spaces including relations of the type (7) as well. At last, summing up all results obtained, we contemplate to show that the temperature must transform under relativistic conditions according to H.Ott and only according to him.

\section{Electric Charge Transfer}

\section{Convection charge transfer}

First, analyze the well-known relation for the convection of the electrical charges

$$
J^{\alpha}=\rho u^{\alpha}, \quad \alpha=0,1,2,3,
$$

where $u^{\alpha}$ is the 4-velocity. Show that (12) is incorrect under the relativistic conditions, since their left and right sides do not transform identically. Indeed, the left side of (12) is Lorentz' invariant if, e.g., $u^{\alpha=1}$ is parallel to $\mathbf{X}_{\mathbf{1}}$ (see Fig.1), but the right side of (12) varies in inverse proportion to $\sqrt{1-\beta^{2}}$. We shall show it below.

In Euclidean space the expression for charge convection

$$
\boldsymbol{j}=\rho \mathbf{v},
$$

is incorrect under relativistic conditions as well, since its left side transforms proportionally to $\sqrt{1-\beta^{2}}$ if, e.g., $\mathbf{v}$ is parallel to $\mathbf{X}_{\mathbf{1}}$, but its right side varies in inverse proportion to $\sqrt{1-\beta^{2}}$ for the observer in the laboratory reference frame, since the velocity $\mathbf{v}$ is independent of $\sqrt{1-\beta^{2}}$.

In Minkowski space $J^{\alpha=1}$ contains 3 quantities: an electric charge $Q[\mathrm{C}]$, an element of area $\Delta \mathrm{S}\left[\mathrm{cm}^{2}\right]$ and time. $Q \neq Q(\mathbf{v}), t=t_{0} / \sqrt{1-\beta^{2}} ; \Delta \mathrm{S}=\Delta \mathrm{S}_{0}$ if the velocity $\mathbf{v}$ is parallel to the axis $\mathbf{X}_{\mathbf{1}}$. Besides, $J^{\alpha=1} \sim \frac{J_{(0)}^{1}}{\sqrt{1-\beta^{2}}}$. Calculating the quantity $J^{\alpha=1}$, the observer at rest in the 
laboratory reference frame should find experimentally the above three quantities. Finally we have

$$
J^{\alpha=1}=J_{0}^{\alpha=1},
$$

since $J^{\alpha=1} \sim \frac{Q}{\Delta S t \sqrt{1-\beta^{2}}} . J_{(0)}^{\alpha=1}$

Now study the right-hand-side of (12). Rewrite (12) as

$$
\boldsymbol{J}=\boldsymbol{J}(\rho \mathbf{u}, i \mathrm{c} \rho),
$$

where $\mathrm{c}$ is the speed of light, $\mathbf{u}$ is the spatial component of the 4 -velocity vector.

For u we have [6]

$$
\mathbf{u}=u=\frac{u^{\prime} \sqrt{1-\beta^{2}}+v\left\{\frac{\left(u^{\prime} v\right)}{c^{2}}\left(\sqrt{1-\beta^{2}}-1\right)+1\right\}}{1+\frac{u^{\prime} v}{c^{2}}},
$$

where $v$ is the velocity vector of the moving reference frame $\mathrm{X}^{\prime}$ relative to the reference one $\mathrm{X}$

We have if $u=u_{x}^{\prime} \| v[6]$ :

$$
u_{x}=\left(u_{x}^{\prime}+v_{x}\right) /\left(1+\frac{u_{x}^{\prime} v_{x}}{c^{2}}\right),
$$

and if $u=u_{y(z)} \perp v$, we have, in turn,

$$
u_{y(z)}=\frac{u_{y(z)}^{\prime} \sqrt{1-\beta^{2}}}{1+\frac{u_{x}^{\prime} v_{x}}{c^{2}}},
$$

where $u_{x}$ is the projection 4-velocity $u$ onto the axis $\mathbf{X}_{1}$, $u_{x}^{\prime}$ is the projection 4-velocity $u^{\prime}$ onto the axis $\mathbf{X}_{1}{ }^{\prime}$, i.e., the projection of the velocity in the moving reference frame.

As seen, the law of velocity composition in Euclidean space does not differ from a similar law in Minkowski space.

Since the volume $V=V_{0} \sqrt{1-\beta^{2}}$, we have, e.g., for the right-hand-side of (12) and (15) for $\alpha=1$ :

$$
\rho u_{\mathrm{x}} \sim \frac{1}{\sqrt{1-\beta^{2}}} .
$$

If $u \perp v$, the product $\rho u_{2(3)}$ is Lorentz invariant like the left-hand side of (12) and (15). Thus expressions (12) and (15) are meaningless.

Consequently, the correct expressions for the convection transfer of electric charges are essentially differ from expressions (12) and (15). Find them.

Suppose that the term in right-hand-side of (13) contains a factor. It is a dimensionless tensor of rank 2 . Find it using the methods represented in [2] by C. Eckart.

We write down this factor in the form

$$
s_{\alpha \beta}=\frac{\Delta l_{\alpha}}{\Delta s} \frac{\Delta l_{\beta}}{\Delta s}, \alpha, \beta=1,2,3,
$$

where $\Delta l_{\alpha(\beta)}$ are the vectors (cm) oriented in space; $s$ is the invariant interval; $\Delta l_{\ldots}=\Delta l_{(0) \ldots} \sqrt{1-\beta^{2}}$, if $l$ is parallel to $\mathbf{v}$. If it is perpendicular to $\mathbf{v}, \Delta l_{\ldots}=\Delta l_{(0)}$. Then (13) we can write as

$$
j_{\alpha}=s_{\alpha \beta} \rho v_{\beta}, s_{\alpha \beta}=0, \text { if } \alpha \neq \beta .
$$

Now the right-hand-side of (13) and (15) will transform like their left-hand-side under the relativistic conditions. Consequently, the expression (21) is a fundamentally new expression for the electrical charge convection under relativistic conditions.

\section{The charge transfer by electrical conductivity. 3-D formalism}

Take formula (7). Is it correct under relativistic conditions?

Let a system be represented in Fig.1. There is an electro-conducting medium in the object under study. The medium is primordially isotropic. There is only an electrostatic force in it, i.e., here and below a magnetic field is absent. We can write down the law (7) in Euclidean space under relativistic conditions as

$$
\begin{gathered}
j_{1}=\sigma_{11} G_{1}+\sigma_{12} G_{2}+\sigma_{13} G_{3}, \\
j_{2}=\sigma_{21} G_{1}+\sigma_{22} G_{2}+\sigma_{23} G_{3}, \\
j_{3}=\sigma_{31} G_{1}+\sigma_{32} G_{2}+\sigma_{33} G_{3} .
\end{gathered}
$$

Are equations (22) correct under these conditions for the observer in the laboratory reference frame?

To answer this question we should answer a question connected with the tensor $\sigma_{\alpha \beta}$.

In Gaussian system of units the tensor has a dimension [s-1]. However the quantity $1 / \mathrm{s}$ is a scalar but we can represent it as a tensor of rank 2 with the help of Kronecker's object $\delta_{\alpha \beta}$. Then we have:

$$
\sigma_{\alpha \beta}=\delta_{\alpha \beta} \sigma,
$$

where

$$
\delta_{\alpha \beta}=\left(\begin{array}{ccc}
1 & 0 & 0 \\
0 & 1 & 0 \\
0 & 0 & 1
\end{array}\right),
$$

$\sigma$ is the absolute value of the quantity $\sigma_{\alpha \beta}$.

Then $\sigma_{\alpha \beta}=0$ if $\alpha \neq \beta$. The above relations (22) are correct under relativistic conditions, since their left- and right sides transform identically. Show it. 
The left side of (22a) varies in proportion with $\sqrt{1-\beta^{2}}$ since $Q \neq Q(\mathbf{v}), t=t_{0} / \sqrt{1-\beta^{2}} ; \Delta \mathrm{S}=\Delta \mathrm{S}_{0}$ (see above). The right side varies similarly, since the component of the electric intensity $E_{x} \neq E_{x}(v)$. The left sides of (22b) and (22c) are independent of the velocity $\mathbf{v}$, since now the element of area is parallel to the above vector and $\Delta \mathrm{S}=\Delta \mathrm{S}_{0}$ $\sqrt{1-\beta^{2}}$. The right sides of (22b) and (22c) are independent of $\mathbf{v}$ as well, since $E_{y}$ and $E_{z}$ vary in inverse proportion to $\sqrt{1-\beta^{2}}$.

If the medium in the system is primordially anisotropic, then equations (22) can become incorrect, since now, there in general, $\sigma_{\alpha \beta} \neq 0$ if $\alpha \neq \beta$. Will relations (22) be correct under relativistic conditions taking into consideration that now we cannot use, e.g., fundamental tensor $g_{\alpha \beta}$ ? They will, if we use the tensor $s_{\alpha}^{\beta}$, i.e., $\sigma_{\alpha}^{\beta}=s_{\alpha}^{\beta} \sigma$ (an affine tensor). We can write down:

$$
\begin{aligned}
\sigma_{\alpha}^{\beta} & =\left(\begin{array}{lll}
s_{1}^{\prime 1} & s_{1}^{\prime 2} & s_{1}^{\prime 3} \\
s_{2}^{\prime 1} & s_{2}^{\prime 2} & s_{2}^{\prime 3} \\
s_{3}^{\prime 1} & s_{3}^{\prime 2} & s_{3}^{\prime 3}
\end{array}\right) \sigma=s_{\alpha}^{\prime \beta} \sigma, \\
\sigma_{\alpha}^{\beta} & =\left(\begin{array}{lll}
L_{1}^{1} & L_{1}^{2} & L_{1}^{3} \\
L_{2}^{1} & L_{2}^{2} & L_{2}^{3} \\
L_{3}^{1} & L_{3}^{2} & L_{3}^{3}
\end{array}\right),
\end{aligned}
$$

where $s_{\alpha}^{\prime \beta}$ are

$$
s_{\beta}^{\prime \alpha}=\frac{\Delta l^{\prime \alpha}}{\Delta s} \frac{\Delta l_{\beta}^{\prime}}{\Delta s}, \quad \alpha, \beta=1,2,3,
$$

$\Delta l^{\prime \alpha}$ and $\Delta l_{\beta}^{\prime}$ are the covariant and contravariant charge paths.

Then relations (22) can be written down in Euclidean space as

$$
\begin{aligned}
& j_{1}=L_{1}^{1} G_{1}+L_{1}^{2} G_{2}+L_{1}^{3} G_{3}, \\
& j_{2}=L_{2}^{1} G_{1}+L_{2}^{2} G_{2}+L_{2}^{3} G_{3}, \\
& j_{3}=L_{3}^{1} G_{1}+L_{3}^{2} G_{2}+L_{3}^{3} G_{3},
\end{aligned}
$$

Analyze formulae (28). First, analyze (28a). The quantity $j_{1} \sim \sqrt{1-\beta^{2}}$ under relativistic conditions, the products $L_{1}^{1} G_{1}, L_{1}^{2} G_{2}, L_{1}^{3} G_{3}$ transforms like $j_{1}$ if $s_{1}^{11} \neq s_{1}^{\prime 1}(v)$, $L_{1}^{1} \sim \sqrt{1-\beta^{2}} \quad G_{1} \neq G_{1}(v), \quad L_{1}^{2(3)} \sim 1-\beta^{2} \quad, \quad$ and $G_{2(3)} \sim 1 / \sqrt{1-\beta^{2}}$.
Now analyze (28b) and (28c). The quantities $j_{2}, j_{3}$ are Lorentz invariants as well as the products $L_{2}^{3} G_{3}$ and $L_{3}^{2} G_{2}$. In turn, the products $L_{2}^{1} G_{1}, L_{3}^{1} G_{1}$ are Lorentz invariants if $L_{2(3)}^{1}$ vary in proportion to $1 / \sqrt{1-\beta^{2}}$. The terms $L_{2}^{3} G_{3}, L_{3}^{2} G_{2}$ are Lorentz invariants as well. Then relations (28) are correct.

Here $L_{\alpha}^{\beta}$ cannot equals to $L_{\beta}^{\alpha}$, i.e., the reciprocity principle cannot be valid under relativistic conditions.

The expressions (22) and (28) are fundamentally new relations for the electrical charge conductivity in Euclidean space under relativistic when the medium of the system under study is primordially anisotropic.

\section{Joule Heat under relativistic conditions}

Will (10) be correct under relativistic conditions (Euclidean space)?

Analyze the formula.

Its left-hand-side varies in inverse proportion to $\sqrt{1-\beta^{2}}$, since heat $Q=Q_{0} / \sqrt{1-\beta^{2}}$ (i.e., according to H.Ott), $\quad V=V_{0} \sqrt{1-\beta^{2}} \quad, \quad t=t_{0} / \sqrt{1-\beta^{2}} ; \quad$ the right-hand-side of (10) varies like its left-hand-side. Indeed, the charge $Q \neq \mathrm{Q}(\mathrm{v}), \Delta \mathrm{S}=\Delta \mathrm{S} 0$ if the vector $\mathrm{v} \perp \Delta \mathrm{S}$ and this quantity varies in proportion to $\sqrt{1-\beta^{2}}$ if $\mathrm{v} \| \Delta \mathrm{S}$; at last, $E_{x}$ is independent of $\sqrt{1-\beta^{2}}$, however, the quantities $E_{y(z)} \sim 1 / \sqrt{1-\beta^{2}}$.

If the heat $Q$ would depend on v according to M.Planck, then the relation (10) were incorrect under relativistic conditions.

\section{The charge transfer by electrical conductivity. 4-D formalism}

Now study the process of electrical conductivity in the 4-formalism. For this case we can write down, taking into consideration, that the process is stationary one, and therefore $E_{0}=0$, since $\frac{\partial \rho}{\partial t}=0$. Then we have, e.g., for $J^{\alpha}$

$$
J^{\alpha}=\Lambda^{\alpha \beta} E_{\beta},
$$

where $J^{\alpha}$ is the contravariant 4-current density, $\Lambda^{\alpha \beta}$ is the contravariant 4-tensor of conductivity; $E_{\beta}$ is the covariant 4-electrical intensity (affine tensors). In fact, (29) is the formula of charge diffusion due to an external force affecting them [4].

Define the quantities $J^{\alpha}$ and $\Lambda^{\alpha \beta}$. 


$$
J^{\alpha}=\frac{Q(\alpha)}{\Delta S(\alpha) t} n^{\alpha} ; n^{\alpha}=\mathrm{n}=\left(\begin{array}{c}
n^{0} \\
n^{1} \\
n^{2} \\
n^{3}
\end{array}\right), \quad \alpha=0,1,2,3
$$

where $Q(\alpha)$ is the number of charges passing through an area element $\Delta S(\alpha)$ in the directions $\alpha$ per unit of time; $n^{\alpha}$ are the components of the dimensionless 4 -vector $\mathbf{n}$ perpendicular to the area elements $\Delta S(\alpha)$, i.e., the latter is perpendicular to the direction $\alpha$.

$$
n^{1} \sim \frac{n_{(0)}^{1}}{\sqrt{1-\beta^{2}}}, n^{2}=n_{(0)}^{2} ; n^{3}=n_{(0)}^{3} .
$$

In turn, we have for $\Lambda_{\alpha \beta}$ :

$$
\Lambda^{\alpha \beta}=\sigma n^{\alpha \beta} / \Delta S(\alpha)=\sigma \Delta l^{\alpha} \Delta l^{\beta} / \Delta S(\alpha),
$$

where $n^{\alpha \beta}$ is the contravariant 4-tensor of order 2; $\Delta l^{\alpha(\beta)}$ is the charge path $(\mathrm{cm})$.

We can write down (29) as

$$
\begin{gathered}
J^{0}=\Lambda^{00} E_{0}+\Lambda^{01} E_{1}+\Lambda^{02} E_{2}+\Lambda^{03} E_{3}=0, \\
J^{1}=\Lambda^{10} E_{0}+\Lambda^{11} E_{1}+\Lambda^{12} E_{2}+\Lambda^{13} E_{3}, \\
J^{2}=\Lambda^{20} E_{0}+\Lambda^{21} E_{1}+\Lambda^{22} E_{2}+\Lambda^{23} E_{3}, \\
J^{3}=\Lambda^{30} E_{0}+\Lambda^{31} E_{1}+\Lambda^{32} E_{2}+\Lambda^{33} E_{3}, \\
\Lambda^{\alpha \beta}=\left(\begin{array}{cccc}
\Lambda^{00} & \Lambda^{01} & \Lambda^{02} & \Lambda^{03} \\
\Lambda^{10} & \Lambda^{11} & \Lambda^{12} & \Lambda^{13} \\
\Lambda^{20} & \Lambda^{21} & \Lambda^{22} & \Lambda^{23} \\
\Lambda^{30} & \Lambda^{31} & \Lambda^{32} & \Lambda^{33}
\end{array}\right)= \\
\left(\begin{array}{cccc}
\Lambda^{00} & 0 & 0 & 0 \\
0 & \Lambda^{11} & \Lambda^{12} & \Lambda^{13} \\
0 & \Lambda^{21} & \Lambda^{22} & \Lambda^{23} \\
0 & \Lambda^{31} & \Lambda^{32} & \Lambda^{33}
\end{array}\right),
\end{gathered}
$$

We have finally

$$
\begin{gathered}
J^{1}=\Lambda^{11} E_{1}+\Lambda^{12} E_{2}+\Lambda^{13} E_{3}, \\
J^{2}=\Lambda^{21} E_{1}+\Lambda^{22} E_{2}+\Lambda^{23} E_{3}, \\
J^{3}=\Lambda^{31} E_{1}+\Lambda^{32} E_{2}+\Lambda^{33} E_{3},
\end{gathered}
$$

$\Lambda^{\alpha \beta}=\Lambda^{\beta \alpha}$ are Onsager's phenomenological coefficients [4].
Analyze formulae (34). First, analyze (34a).

The left-hand-side of (34a) is Lorentz invariant. Indeed, $t \sim 1 / \sqrt{1-\beta^{2}}, \Delta S(\alpha=1)=\Delta S_{(0)}(\alpha=1), Q \neq Q(\mathrm{v})$ (see (30) and (31)). In turn, all terms of the right-hand-sides of (34a) are also Lorentz invariants, since $n^{11} \sim\left(1-\beta^{2}\right)^{-1} ; n^{12(13)}=n^{21(31)} \sim 1 / \sqrt{1-\beta^{2}}$ (see (32)) and

$$
E_{1} \sim E_{1}^{(0)} \sqrt{1-\beta^{2}} ; E_{2(3)}=E_{2(3)}^{(0)} ; E_{1(2,3)} E^{1(2,3)}=1,
$$

Now analyze (34b) and (34c).

Their left-hand- and right-hand-sides are Lorentz's invariants as well, since $\Delta S(\alpha=2,3)=\Delta S_{(0)}(\alpha=2,3) \sqrt{1-\beta^{2}}$ $n^{22}=n_{(0)}^{22} ; n^{33}=n_{(0)}^{33} ; n^{23}=n^{32}=n_{(0)}^{23}=n_{(0)}^{32}$.

Finally, solving the problem, we may use, instead of the expression (29), the following dependence:

$$
J_{\gamma}=\Lambda_{\gamma \delta} E^{\delta} .
$$

We shall use this expression below. Here we should also note the following. $J_{\gamma} \neq J_{\alpha}$ and $j_{\gamma} \neq j_{\alpha}$, however, $E^{\beta}=E^{\delta} ; G^{\beta}=G^{\delta}$ and $E_{\beta}=E_{\delta} ; G_{\beta}=G_{\delta}$, since the electrical intensities are pure vectors but the quantities $J, j$ and $\Lambda, L$ are not the pure ones. For example, $\Lambda^{\alpha \beta}$ is the tensor $n^{\alpha \beta}$ multiplied by scalars $\sigma\left[t^{-1}\right]$ and $1 / \Delta S(\alpha)$. In turn, $J^{\alpha}$ is the vector obtained by tensor multiplication of the tensor $\Lambda^{\alpha \beta}$ and the vector $E_{\beta}$ with the consequent contracture on the indices $\beta$.

Now find a connection between the electrical intensity $E^{\beta}, E_{\beta}$ in Minkowski space and the electrical ones $G^{\beta}, G_{\beta}$ in Euclidean space where the medium is primordially isotropic. First, represent, for this purpose, expressions (7), (29) and (36) as

$$
\begin{gathered}
j_{\gamma}=L_{\gamma \delta} G^{\delta}=\sigma g_{\gamma \delta} G^{\delta} ; G_{\gamma}=g_{\gamma \delta} G^{\delta}, \\
j^{\alpha}=L^{\alpha \beta} G_{\beta}=\sigma g^{\alpha \beta} G_{\beta} ; G^{\alpha}=g^{\alpha \beta} G_{\beta} ; \\
J_{\gamma}=\Lambda_{\gamma \delta} E^{\delta}=\sigma g_{\gamma \delta} E^{\delta} ; E_{\gamma}=g_{\gamma \delta} E^{\delta}, \\
J^{\alpha}=\Lambda^{\alpha \beta} E_{\beta}=\sigma g^{\alpha \beta} E_{\beta} ; E^{\alpha}=g^{\alpha \beta} E_{\beta},
\end{gathered}
$$

where $g_{\alpha \beta}, g^{\alpha \beta}, g_{\gamma \delta} . g^{\gamma \delta}$ are the fundamental tensors;

$$
\begin{array}{r}
J^{\alpha(\gamma)}=j^{\alpha(\gamma)} ; J_{\alpha(\gamma)}=j_{\alpha(\gamma)}, \text { and } \\
J_{\alpha}=\frac{Q(\alpha)}{\Delta S(\alpha) t} n_{\alpha},
\end{array}
$$




$$
\begin{gathered}
J_{\gamma}=\frac{Q(\gamma)}{\Delta S(\gamma) t} n_{\gamma} ; \\
n_{1} \sim n_{(0) 1} \sqrt{1-\beta^{2}} ; n_{2}=n_{(0) 2} ; n_{3}=n_{(0) 3},
\end{gathered}
$$

see (30) - (31). 40):

$$
\begin{aligned}
& J^{1}=J_{(0)}^{1} \sim \sqrt{1-\beta^{2}} E^{1}=\sqrt{1-\beta^{2}} \frac{E_{(0)}^{1}}{\sqrt{1-\beta^{2}}} ; \\
& j^{1}=j_{(0)}^{1} \sim \sqrt{1-\beta^{2}} G^{1} C=\sqrt{1-\beta^{2}} G_{0}^{1} \frac{1}{\sqrt{1-\beta^{2}}},
\end{aligned}
$$

and if $J^{\alpha}=j^{\alpha}$, then

$$
\begin{gathered}
E^{1}=\frac{G^{1}}{\sqrt{1-\beta^{2}}} ; \\
J_{1}=J_{(0) 1}\left(1-\beta^{2}\right) \sim \sqrt{1-\beta^{2}} E_{1}=\sqrt{1-\beta^{2}} E_{(0) 1} \sqrt{1-\beta^{2}} ; \\
j_{1}=j_{(0) 1} \sim \frac{\sqrt{1-\beta^{2}}}{C} G_{1}=\left(1-\beta^{2}\right) G_{(0) 1},
\end{gathered}
$$

and if $J_{\gamma}=j_{\gamma}$, then

$$
G_{1}=\frac{E_{1}}{\sqrt{1-\beta^{2}}}
$$

where $C=1 / \sqrt{1-\beta^{2}}$ is the constant quantity involved in (44) and (46) according Curie-Veitsman's generalized principle $[8,9]$.

Further we have:

$$
\begin{aligned}
& J^{2}=J^{(0) 2} \sim \sqrt{1-\beta^{2}} E^{(0) 2} ; \\
& j^{2}=j^{(0) 2} \sim \sqrt{1-\beta^{2}} G^{2(3)}=\sqrt{1-\beta^{2}} \frac{G^{(0) 2}}{\sqrt{1-\beta^{2}}},
\end{aligned}
$$

and if $J^{\alpha}=j^{\alpha}$, then

$$
\begin{gathered}
G^{2(3)}=\frac{E^{2(3)}}{\sqrt{1-\beta^{2}}} ; \\
J_{2}=J_{(0) 2} \sim \sqrt{1-\beta^{2}} E_{(0) 2} ; \\
j_{2}=j_{(0) 2} \sim \sqrt{1-\beta^{2}} G_{2}=\left(1-\beta^{2}\right) G_{(0) 2} C^{2},
\end{gathered}
$$

where $C=1 / \sqrt{1-\beta^{2}}$ (see above), and if $J_{\gamma}=j_{\gamma}$, then

$$
E_{2}=\frac{G_{2}}{\sqrt{1-\beta^{2}}}
$$

the similar result we have for $E_{3}, G_{3}$ :

$$
E_{3}=\frac{G_{3}}{\sqrt{1-\beta^{2}}}
$$

There are the following dependences in SR (Euclidean vectors)[7]:

$$
\begin{gathered}
E_{\alpha}=\frac{G_{\alpha}}{\sqrt{1-\beta^{2}}} ; \alpha=1,2,3, \\
G_{\alpha}=\frac{E_{\alpha}}{\sqrt{1-\beta^{2}}} ; \alpha=1,2,3 ;
\end{gathered}
$$

formula (52) is correct for an observer at rest in the laboratory reference frame; formula (53) is correct for an observer in a reference frame, moving with the velocity $v$, relative to the laboratory reference frame. Formulae (44), (50), (51) and (46), (48) are in full accordance with SR.

The expressions (29), (34) and (36) are fundamentally new relations for the electrical charge conductivity in Minkowski space under relativistic conditions when the medium of the system under study is primordially anisotropic.

\section{Heat Transfer}

We have concerned above the problem of heat transfer under relativistic conditions in Euclidean space for the medium being primordially isotropic [1]. Now we shall examine the heat transfer in Euclidean and Minkowski spaces under relativistic conditions when the medium is primordially anisotropic. For the heat transfer in Euclidean space we can write down $[1,4]$ :

$$
\begin{aligned}
& I_{1}^{(\lambda)}=-D_{11}^{(\lambda)} \frac{\partial q}{\partial x_{1}}-D_{12}^{(\lambda)} \frac{\partial q}{\partial x_{2}}-D_{13}^{(\lambda)} \frac{\partial q}{\partial x_{3}}, \\
& I_{2}^{(\lambda)}=-D_{21}^{(\lambda)} \frac{\partial q}{\partial x_{1}}-D_{22}^{(\lambda)} \frac{\partial q}{\partial x_{2}}-D_{23}^{(\lambda)} \frac{\partial q}{\partial x_{3}}, \\
& I_{3}^{(\lambda)}=-D_{31}^{(\lambda)} \frac{\partial q}{\partial x_{1}}-D_{32}^{(\lambda)} \frac{\partial q}{\partial x_{2}}-D_{33}^{(\lambda)} \frac{\partial q}{\partial x_{3}} .
\end{aligned}
$$

for $D_{i j}^{(\lambda)}\left(\mathrm{cm}^{2} \cdot \mathrm{s}^{-1}\right)$ (the heat conductivity coefficient) which may be non-zero if $i \neq j$.

In this case the terms on the left- and right-hand-sides of equations (54) transform identically under relativistic conditions since, the cross diffusion coefficients are tensors, their linear components (c) are perpendicular to one another.

It means that, e.g., heat gradient $\frac{\partial q}{\partial x_{2}}$ generates simultaneously heat fluxes in two directions relative to the coordinate axes.

Take, e.g., crystals of trigonal (dolomite, sodium periodate) and tetragonal (scheelite) systems. The tensors of the heat 
transfer coefficients for these systems take the form [4]

$$
\left(\begin{array}{ccc}
D_{11} & D_{12} & 0 \\
-D_{12} & D_{11} & 0 \\
0 & 0 & D_{33}
\end{array}\right) .
$$

The experiments showed that $D_{12}=D_{21}=-D_{12}=0$.

There are, in turn, the following tensor coefficients $D_{i j}^{(\lambda)}$ for the triclinic and monoclinic crystal systems

$$
\begin{aligned}
& \left(\begin{array}{ccc}
D_{11} & D_{12} & D_{13} \\
D_{21} & D_{22} & D_{23} \\
D_{31} & D_{32} & D_{33}
\end{array}\right), \\
& \left(\begin{array}{ccc}
D_{11} & D_{12} & 0 \\
D_{21} & D_{22} & 0 \\
0 & 0 & D_{33}
\end{array}\right),
\end{aligned}
$$

The coefficients $D_{i j}^{(\lambda)}$ may be non-zero if $i \neq j$.

Will the relations (54) be correct under Lorentz transformations? They will, since their left- and right-hand-sides transform identically under relativistic conditions. Here we take into consideration that $Q=Q_{0} / \sqrt{1-\beta^{2}}, q=q_{0} /\left(1-\beta^{2}\right), t=t_{0} / \sqrt{1-\beta^{2}}$, $S=S_{0} \quad$ if vector $\quad \mathrm{v}$ is perpendicular to $S_{0}$, $S=S_{0} \sqrt{1-\beta^{2}}$ if one side of an element of the area is parallel to $\mathrm{v}, V=V_{0} \sqrt{1-\beta^{2}}, D_{11}^{(\lambda)}=D_{011}^{(\lambda)} \sqrt{\left(1-\beta^{2}\right)^{3}}$, $D_{22}^{(\lambda)}=D_{022}^{(\lambda)} \sqrt{1-\beta^{2}}, D_{33}^{(\lambda)}=D_{033}^{(\lambda)} \sqrt{1-\beta^{2}}$,

$$
\begin{gathered}
D_{12}^{(\lambda)}=D_{21}^{(\lambda)}=D_{012(21)}^{(\lambda)}\left(1-\beta^{2}\right), \\
D_{13}^{(\lambda)}=D_{31}^{(\lambda)}=D_{013(31)}^{(\lambda)}\left(1-\beta^{2}\right) \\
D_{23}^{(\lambda)}=D_{32}^{(\lambda)}=D_{023(32)}^{(\lambda)} \sqrt{1-\beta^{2}} .
\end{gathered}
$$

It should also be. Relations (54) will incorrect if the condition $T=T_{0} / \sqrt{1-\beta^{2}}$ is not satisfied, since incidentally the above condition $Q=Q_{0} / \sqrt{1-\beta^{2}}$ and the condition $S=S_{0}$ (here $\mathrm{S}$ is the entropy) are violated as well.

In Minkowski space we can write down for the heat transfer under relativistic conditions using affine tensors:

$$
I_{(\lambda)}^{\alpha 1}=-D_{(\lambda)}^{\alpha \beta} \frac{\partial q}{\partial x^{\beta}}, \alpha, \beta=0,1,2.3,
$$

For a stationary process, as $\partial q / \partial x^{0}=0$, we have

$$
\begin{gathered}
I_{(\lambda)}^{1}=-D_{(\lambda)}^{11} \frac{\partial q}{\partial x^{1}}-D_{(\lambda)}^{12} \frac{\partial q}{\partial x^{2}}-D_{(\lambda)}^{13} \frac{\partial q}{\partial x^{3}} \\
I_{(\lambda)}^{2}=-D_{(\lambda)}^{21} \frac{\partial q}{\partial x^{1}}-D_{(\lambda)}^{22} \frac{\partial q}{\partial x^{2}}-D_{(\lambda)}^{23} \frac{\partial q}{\partial x^{3}} \\
I_{(\lambda)}^{3}=-D_{(\lambda)}^{31} \frac{\partial q}{\partial x^{1}}-D_{(\lambda)}^{32} \frac{\partial q}{\partial x^{2}}-D_{(\lambda)}^{33} \frac{\partial q}{\partial x^{3}}
\end{gathered}
$$

where $I_{(\lambda)}^{\alpha}$ is the heat flux vector; $D_{(\lambda)}^{\varepsilon \beta}$ is the heat flux coefficient - contravariant tensor of rank 2.

The vector $I_{(\lambda)}^{\alpha}$ contains scalar and vector parts. The scalar one includes heat $\mathrm{Q}(\mathrm{J})$, time $\mathrm{t}(\mathrm{s})$ and an area element $\Delta \mathrm{S}(\alpha ; \mathrm{cm} 2)$ oriented in space, i.e., $Q / t \Delta \mathrm{S}(\alpha)$. The vector part $\mathbf{i}^{\alpha}$ is a contravariant dimensionless unit-vector (under normal conditions $\mathbf{i}_{0}^{\alpha}$ ); it is perpendicular to the corresponding area element $\Delta \mathrm{S}(\alpha)$. Then

$$
\begin{gathered}
I_{(\lambda)}^{\alpha} \equiv[Q / t \Delta \mathrm{S}(\alpha)] \mathbf{i}^{\alpha}, \\
\mathrm{i}^{\alpha}=\mathrm{i}_{0}^{\alpha} / \sqrt{1-\beta^{2}} .
\end{gathered}
$$

if $\alpha=1$, and

$$
\mathrm{i}^{\alpha}=\mathrm{i}_{0}^{\alpha},
$$

if $\alpha=2,3$.

Then $I_{(\lambda)}^{\alpha}$ varies in inverse proportion to $\sqrt{1-\beta^{2}}$.

The coefficients $D_{(\lambda)}^{\alpha \beta}$ also contain two parts: scalar and tensor ones. The scalar part includes time $\mathrm{t}$ and linear sizes $\Delta l(\alpha)$ and $\Delta l(\beta)$ oriented in the directions $\alpha, \beta=1,2,3 . \quad \Delta l=\Delta l_{0} \sqrt{1-\beta^{2}}, \quad$ if $\quad \Delta l \quad \| \mathrm{v} \quad$ and $\Delta l=\Delta l_{0}$, if $\Delta l \perp \mathrm{v}$. The tensor part $\Delta l^{\alpha}, \Delta l^{\beta}$ is the contravariant vectors. If $\Delta l^{\alpha(\beta)} \| \mathrm{v}$, then $\Delta l^{\alpha(\beta)} \sim$ $\frac{\Delta l_{0}^{\alpha(\beta)}}{\sqrt{1-\beta^{2}}} ; \Delta l^{\alpha(\beta)} \perp \mathrm{v}$,

then $\Delta l^{\alpha(\beta)}=\Delta l_{0}^{\alpha(\beta)}$.

The gradients of the quantity $q$ also contain two parts: the scalar and vector ones. The scalar part is a derivative of $q$ with respect to $x^{\beta}$, i.e. $\lim \frac{\Delta q}{\Delta x^{\beta}}$, as $\Delta x^{\beta} \rightarrow 0 . q=\frac{\delta Q}{\delta V}$ $\left(\mathrm{J} \cdot \mathrm{cm}^{-3}\right)$. The vector part in the right-hand-side of (57) is a covariant vector $i_{\beta}$. If $i_{\beta} \| \mathbf{v}$,

then

If $\mathrm{i}_{\beta} \perp \mathrm{v}$, then

$$
\mathrm{i}_{\beta} \sim \mathrm{i}_{(0) \beta} \sqrt{1-\beta^{2}} .
$$

$$
\mathrm{i}_{\beta}=\mathrm{i}_{(0) \beta} \quad .
$$


Contracting the indices $\beta$ in the right-hand-side of (57), we bring up that all terms vary in inverse proportion to $\sqrt{1-\beta^{2}}$, i.e., the solutions (57) are correct.

The expressions (54) and (37) are fundamentally new relations for the heat transfer in Euclidean and Minkowski spaces under relativistic conditions when the medium of the system under study is primordially anisotropic.

\section{Special Case of Conductivity (4-D Formalism)}

Here we concern a special case of conductivity in Minkowski space for an anisotropic and dense medium, incidentally, its refractory index $n \neq 1$. Then it is possible for electrons $v>\frac{c}{n}$, moving with velocities where $\sqrt{1-\frac{(v n)^{2}}{c^{2}}}$ is an imaginary number, i.e. Cherenkov's case takes place. However, the dependences (34) are correct under these conditions, since all imaginary units cancel in terms of relations (34) mutually. Take, e.g., the quantity

$$
J^{\alpha}=\frac{Q(\alpha)}{\Delta S(\alpha) t} n^{\alpha} ; \alpha=1,
$$

(see the relations (30) above). It is a real number, since $n^{\alpha=1}$ and $t$ vary in inverse proportion to $\sqrt{1-\frac{(v n)^{2}}{c^{2}}}$ under these conditions.

\section{Discussion}

As shown in Introduction, the well-known dependences for charge and heat transfer, i.e., formulae (10), (3) and (14), are incorrect under relativistic conditions so as, first, their left- and right-hand-sides do not transform identically in the case under study; second, the kinetic coefficients in the formulae are scalars under the Lorentz transformations; third, the temperature does not transform under the Lorentz ones. It is independent of the velocity of the moving object. The formulae (10), (21), (28) and (29) obtained by us are fully correct under relativistic conditions. As $v \rightarrow c$, their leftand right-hand-sides transform identically and kinetic coefficients in the formulae are tensors of rank 2 . The medium in the object can be primordially isotropic and anisotropic. As to the temperature, so it is present in a implicit form in the relation (10), (54), (57) and (58) and transforms there according to H.Ott. Today the transformation of the temperature under relativistic condition is the key problem of relativistic thermodynamics.

Having obtained the above results, we can now make important conclusions concerning relativistic thermodynamics as a whole. First of all, they concern a relativistic temperature $T$. Taking into consideration the above results and results obtained in [1, 5, 8, 9 - 17], we can now contend with a high degree of probability: only the dependence of the kind $T \sim 1 / \sqrt{1-\beta^{2}}$ provides a possibility of obtaining a consistent relativistic thermodynamics, which is correct over the entire interval 0 $c$ of the motion velocities of the object under study. As a proof of this argument we represent all fundamental thermodynamical parameters and dependences (as already known and obtained recently) containing them. There is the temperature in these parameters and dependences. It varies in inverse proportion to $\sqrt{1-\beta^{2}}$.

1.The average value of kinetic energy of the molecule (atom) translational motion:

$$
\bar{\varepsilon}=\frac{3}{2} k T .
$$

Evidently, the dependence (63) is correct over the entire interval of object motion velocities if the temperature varies in inverse proportion to $\sqrt{1-\beta^{2}}$.

2. The equation of state of perfect gases $[1,11]$ :

$$
\frac{p V}{1-\beta^{2}}=N k T
$$

naturally, for the observer in the laboratory reference frame; the pressure $p$ is Lorentz invariant; the volume of gas $V=V_{0} \sqrt{1-\beta^{2}}$.

3. The equations of state for the interface separating a pure liquid and its vapours $[1,11]$.

The equations are valid over the entire interval $0-\mathbf{v}$ of the object velocities if $T=T_{0} / \sqrt{1-\beta^{2}}$; besides, the surface tension is Lorentz invariant like the pressure,

4. The thermodynamic potentials (internal energy, enthalpy, free energy, free enthalpy) including their specific $\left(\mathrm{J} / \mathrm{cm}^{2}\right)$ values [14]

Dependences obtained are correct on the entire velocity interval $0-\mathbf{v}$ if $T=T_{0} / \sqrt{1-\beta^{2}}$; the dependences are not contradictory and absurdity. A similar result is obtained for the chemical potential (including its specific values), i.e., $\mu \sim 1 / \sqrt{1-\beta^{2}}[13,15]$.

5. Small fluctuations of volume, microparticles, temperature [12].

The dependences obtained are valid for intervals of object velocities where fluctuations are small. If the temperature in the formulae obtained varies in inverse proportion to $\sqrt{1-\beta^{2}}$, we do not come to any contradictions or absurdity.

6. Stefan-Boltzmann's law under relativistic conditions $[16,17]$. 
In $[16,17]$ Veitsman obtained dependences connecting the black-body radiation and the absolute temperature under relativistic conditions. The dependences can be correct if only $T=T_{0} / \sqrt{1-\beta^{2}}$.

7. The theory of the charge transfer according to H.Ott [5].

The theory is correct under relativistic conditions if only the object temperature varies in inverse proportion to $\sqrt{1-\beta^{2}}$ (see above).

8. A closed thermodynamical cycle and the well-known thermodynamical principles as follows

$$
\begin{gathered}
\oint \delta E=0, \\
\oint \delta S=\oint \frac{\delta Q}{T}=0,
\end{gathered}
$$

are correct if $T=T_{0} / \sqrt{1-\beta^{2}}[5,12]$.

9. The radiation energy and momentum vary in the range $0-$ $c$ inverse proportional to $\sqrt{1-\beta^{2}}$, i.e., as $T=T_{0} / \sqrt{1-\beta^{2}}$ [5].

10. Chemical reaction rates $w$, e.g.,

$$
w=\frac{1}{v_{\mathrm{A}}} \frac{d[\mathrm{~A}]}{d t}=a A_{r}=(a R T) \frac{A}{R T}=a_{1} A_{1},
$$

vary over the interval $0-c$ inverse proportional to $\sqrt{1-\beta^{2}}$, i.e., as $T=T_{0} / \sqrt{1-\beta^{2}}[13]$. Here $v_{\mathrm{A}}$ is the stoichiometric coefficients of the substance $\mathrm{A} ; A_{r}$ is the affinity according to De Donde; $a$ is a phenomenological coefficient.

We cannot obtain a consistent aggregate of the thermodynamic parameters and dependences containing the parameters under Lorentz transformation if $T=T_{0}$ and

$T=T_{0} \sqrt{1-\beta^{2}}$. As to the theories where the latter temperature transformations are used for a specific process, and contradictions are absent at first sight, so we again come to absurdity but in a implicit form. For example, Clapeyron's equation $p V=N k T$ is quite formally correct under relativistic conditions even if the gas temperature varies according to Planck, however at the same time formula (63) describing the energy of the molecule (atom) translational moving is correct under the relativistic conditions if the temperature of the object transforms only following Ott's theory. The other variants are absent here. However this dependence and Clapeyron's equation treat the same division of physics - thermodynamics of gases and vapours. But if in the framework of this division of physics the temperature of the system may transform under relativistic conditions otherwise, then it is nonsense.

\section{Conclusions}

1. The relations were obtained for the electrical charge transfer under relativistic conditions when the processes were close to equilibrium; the following processes were studied:

a. electrical charge convection (Eq.(21));

b. charge transfer by electrical conductivity allowing for primordial substance anisotropy (3-D and 4-D formalism; Eqs (22) and (27));

c. the above relations (22) and (27) are correct for the case when the velocity of electrons in the medium under study can be more than the velocity of light (the Cherenkov case).

2. The relations were obtained (Eqs. (54) and (57)) for the heat transfer under relativistic conditions (3-D and 4-D formalism) when the process was close to equilibrium and the medium primordially anisotropic.

3. It was shown that the temperature under relativistic conditions had to transform according to H.Ott exclusively, i.e., $T=T_{0} / \sqrt{1-\beta^{2}}$.

\section{REFERENCES}

[1] E.V. Veitsman. Some Problems in Relativistic Thermodynamics. J. Experimental and Theoretical Phys (Russia), Vol.105, №5,1057 - 1067,2007.

[2] C. Eckart. The Thermodynamics of Irreversible Processes. 3. Relativistic Theory of the Simple Fluid. Phys. Rev.,Vol. 58, №11, 919 - 924,1940.

[3] L.D.Landau and E.M.Lifshitz. Theoretical Physics, Hydrodynamics, V.6, §136. Nauka, The Chief Editorial Board of Physical and Mathematical Literature, Moscow, 1986.

[4] R.Haase. Thermodynamik der irreversiblen Prozesse. Dr. Dietrich Steinkopf Verlag, Darmstadt, 1963.

[5] H.Ott. Lorenz-Transformation der Waerme and der Temperatur. Zeitschrift f. Phys. Band 175, Heft 1, 70 - 104, 1963.

[6] E.Madelung. Die matematischen Hilfsmittel des Physikers. Springer-Verlag, Berlin-Goettingen-Heidelberg, 1957.

[7] V.G.Levich. The Course of Theoretical Physics, V.1. Fizmatgiz, Moscow, 1962.

[8] E.V.Veitsman. Interrelation between Thermodynamical Processes in Moving System. Technical Phys.Vol.55, №5, $595-600,2010$.

[9] E.V.Veitsman. Irreversible Process Thermodynamics of Continuous Systems under Relativistic Conditions. Galilean Electrodynamics. Vol. 23, №2, 23 - 32, 2012.

[10] E.V.Veitsman. On Relativistic Surface Tension. Colloid and Int. Sci. Vol. 265, №1, 174 - 178, 2003.

[11] E.V.Veitsman. Relativistic State Equation for the Surface. Colloid and Int. Sci., Vol.290, №1, 231 - 234, 2005 and Corrigendum's, Vol.295, 592, 2006; Vol.333, 411, 2009.

[12] E.V.Veitsman. Temperature and Small Fluctuations in a 
Velocity Interval of a Moving Medium, Galilean Electrodynamics, Vol.23, №2, 23 - 32,2015.

[13] E.V.Veitsman. On the Bond between Chemical Reaction Rate and the Velocity of the System under Study. Am. J. Mod. Phys., Vol.2, №2, 93 - 97,2013.

[14] E.V.Veitsman. Specific Thermodynamical Potential on Surfaces under Relativistic Conditions. J. Colloid Interface Sci.,Vol.337, №2, 355 - 357,2009.
[15] E.V.Veitsman. On the Rate of Relativistic Surface Chemical Reactions. J. Colloid Interface Sci., Vol.275, №2, 555 - 559, 2004.

[16] E.V.Veitsman. Stefan-Boltzmann's Law under Relativistic Conditions. Physical Rev. \& Research International. Vol.4, №1, $217-230,2014$.

[17] E.V.Veitsman. Stefan-Boltzmann's Law under Relativistic Conditions; Generalized Case. Universal J. of Phys. Appl.,Vol.1, №4, 380 - 391, 2013. 